\title{
Inhibitory effect of naphthoquine phosphate against Babesia gibsoni in vitro and Babesia rodhaini in vivo
}

\section{Shengwei Ji}

National Research center for Protozoan diseases, Obihiro University of agriculture and veterinary medicine

\section{Mingming Liu}

Department of Microbiology and immunology, School of Basic Medicine, Hubei University of Arts and

Science

\section{Eloiza May Galon}

National Research Center for Protozoan Diseases, Obihiro University of Agriculture and Veterinary Medicine

\section{Mohamed Abdo Rizk}

Department of Internal Medicine and Infectious Diseases, Faculty of Veterinary Medicine, Mansoura University

\section{Bumduuren Tuvshintulga}

National research center for protozoan diseases, obihiro university of agriculture and veterinary medicine

Jixu Li

College of Agriculture and Animal science,Qinghai university

Iqra Zafar

National Research Center for Protozoan Diseases, Obihiro University of agriculture and veterinary medicine

\section{Yae Hasegawa}

National research center for Protozoan Diseases,Obihiro University of Agriculture and veterinary medicine

\section{Naoaki Yokoyama}

National Center for Protozoan Diseases, Obihiro University of Agriculture and veterinary medicine Xuenan Xuan ( $\sim$ gen@obihiro.ac.jp )

National Research Center for Protozoan Diseases, Obihiro University of Agriculture and Veterinary Medicine

\section{Research Article}

Keywords: Apicomplexan, naphthoquine, Babesia, babesiosis, treatment 
Posted Date: October 27th, 2021

DOI: https://doi.org/10.21203/rs.3.rs-1011892/v1

License: (c) (i) This work is licensed under a Creative Commons Attribution 4.0 International License. Read Full License

Version of Record: A version of this preprint was published at Parasites \& Vectors on January 7th, 2022. See the published version at https://doi.org/10.1186/s13071-021-05127-0. 


\section{Abstract}

Background: Drug resistance and severe side effects are major challenges in the treatment of babesiosis as they lead to less choices for treatment. Development of new drugs to enrich the treatment strategies and delay the emergence of drug resistance in parasites is still needed. Naphthoquine (NQ) combined with artemisinin treats Plasmodium infection by rapid parasite clearance. The current study repurposed NQ as a babesiosis drug treatment by evaluating the effects of naphthoquine phosphate (NQP) as a single dose treatment for babesiosis.

Methods: In vitro anti-Babesia activity of NQP was tested on Babesia gibsoni cultures. The inhibition of parasite growth was verified using a SYBR green I-based fluorescence assay. In vivo efficacy of NQP was evaluated using BALB/c mice infected with Babesia rodhaini. The parasitemia level and hematocrit values were monitored.

Results: The half maximal inhibitory concentration of NQP against $B$. gibsoni in vitro was $3.3 \pm 0.5 \mu \mathrm{M}$. Oral administration of NQP for 5 successive days at a dose of $40 \mathrm{mg} / \mathrm{kg}$ of body weight resulted in significant inhibition on parasite growth compared with the control group. All mice in NQP-treated group survived, whereas the mice in control group died between days 6 and 9 post infection.

Conclusion: This is the first study to evaluate the anti-Babesia activity of NQP in vitro and in vivo. The results showed that NQP is a promising drug for babesiosis treatment and drug repurposing may provide new treatment strategies for babesiosis.

\section{Background}

Babesiosis is an infectious disease caused by intraerythrocytic parasites of the genus Babesia. More than 100 Babesia species have been identified. The most notable species include $B$. bigemina, $B$. divergens, and $B$. bovis for bovine, $B$. caballi for equine, $B$. canis and $B$. gibsoni for canine, and $B$. microti and $B$. rodhaini for murine [1]. Moreover, several Babesia spp. have been reported to infect humans. Because of its wide range of hosts, babesiosis is one of the most ubiquitous infections of free-living animals and is gaining increasing interest as an emerging zoonosis in humans [1]. The parasites replicate in the mammalian host red blood cells which produce clinical symptoms including fever, hemolytic anemia, anorexia, hemoglobinuria, and emaciation and the severe infection phase often results in death [2]. Current treatment strategies for babesiosis are limited. For instance, imidocarb dipropionate and diminazene aceturate are used for babesiosis in animals, whereas atovaquone combined with azithromycin and clindamycin combined with quinine are used for humans [3]. However, the indiscriminate use and lack of alternative drugs contributed to the emergence of drug resistance in parasites, and reports of adverse side effects are well documented [4, 5].

In the past five decades, the cases of human babesiosis have increased in the United States [6]. A review shows that during 1982 to 1993, 139 hospitalizations occurred due to Babesia infection. Among the patients, 25\% required intensive care stays and 9 patients died [7]. Moreover, a recent babesiosis 
surveillance in the U.S. showed that a total of 7,612 cases of babesiosis were reported to the CDC from 2011 to 2015 . Among the cases, $82.5 \%$ were classified by the reporting health jurisdiction as confirmed and $17.5 \%$ as probable. A total of 7 deaths were attributed to babesiosis and 4 deaths were not babesiosis-related, whereas whether the 35 patients' death were caused by babesiosis were not verified [8]. Atovaquone combined with azithromycin as the standard therapy for babesiosis have reported failures in some clinical cases due to mutations in the cytochrome $b$ gene [5]. The atovaquoneazithromycin drug combination is also effective for canine B. gibsoni infections. However, a recent study documented the increased detection of a cytochrome $b$ gene variant associated with atovaquone resistance in B. gibsoni population in dogs in Japan [9].

Although several promising drugs have been reported for babesiosis, such as tafenoquine (TAF) [10, 11], clofazimine [12], and pyronaridine tetraphosphate [13], but due to geographical restrictions, the development of new drugs to enrich the treatment strategies and delay the emergence of drug resistance is still needed.

Naphthoquine (NQ) is an antimalarial drug that was first synthesized in China in 1986 and registered as naphthoquine phosphate (NQP) in 1993. Initial clinical trials showed NQ monotherapy to be highly efficacious without documented toxicity $[14,15]$. Subsequently, a drug combination comprised of NQ and artemisinin (ART) at a fixed ratio of 1:2.5 was developed in order to retain the strongpoint of the two drugs and delay the possible emergence of drug resistance. Safety data of NQ-containing therapies involving more than 4000 patients recorded no serious adverse experience, hematology, and biochemistry changes. It has been considered as a promising anti-malarial drug candidate and is marketed under the name of $A R C O^{\circledR}$ in various tropical countries [14-16]. Due to the Plasmodium and Babesia genera being closely related, we were interested if NQP has effects against the Babesia parasite. This study aimed to investigate the inhibitory effects of NQP against in vitro cultured Babesia gibsoni, a causative agent of canine babesiosis and in vivo propagated Babesia rodhaini, a highly pathogenic rodent Babesia species.

\section{Methods}

\section{Chemical reagents}

Naphthoquine phosphate (NQP) was purchased from ChemScene (NJ, USA). Tafenoquine (TAF) were purchased from Sigma-Aldrich (Tokyo, Japan). SYBR Green I (SG1) nucleic acid stain was purchased from Lonza America (GA, USA). NQP and TAF were dissolved in dimethyl sulfoxide (DMSO) to prepare a stock solution with the concentration of $40 \mathrm{mg} / \mathrm{ml}$ and diluted with Milli-Q water before use.

\section{Maintenance of the parasites and mouse infection}

In this study, we chose in vitro cultured $B$. gibsoni as a model to evaluate drug effects on the Babesia parasite as it was established and utilized previously in our laboratory [17]. Babesia gibsoni Oita strain [18] was cultured and used for the in vitro study. Briefly, the parasite was grown using canine red 
blood cells (RBCs) suspended in RPMI-1640 supplemented with $20 \%$ canine serum and cultured in 24well plates. For the in vivo evaluation, $B$. rodhaini Australia strain [19] was recovered from the stock in our laboratory. For the maintenance of $B$. rodhaini, cryopreserved parasitized-RBCs were passaged by intraperitoneal (i.p.) injection of mice. Challenge infection was performed with i.p. inoculation of $10^{7}$ fresh $B$. rodhaini infected RBCs (iRBCs). A total of 20 BALB/c mice (6-weeks old) were purchased from CLEA Japan and were used to maintain $B$. rodhaini for the in vivo study.

\section{In vitro B. gibsoni growth inhibition assay}

To test the activity of NQP against B. gibsoni, SYBR Green 囚-based fluorescence assay was performed as previously reported [20]. Briefly, in vitro cultures of $B$. gibsoni were diluted to $1 \%$ parasitemia with fresh canine RBCs. The NQP was dissolved with medium at concentrations that ranged from 0.1 to $5 \mu \mathrm{M}$, and incubated with infected RBCs (iRBC) in triplicate in 96-well plates with $5 \%$ hematocrit for 96 hours. The fluorescence values were evaluated using the fluorescence spectrophotometer (485 and $518 \mathrm{~nm}$, Fluoroskan Ascent, USA) and the inhibition activity and half inhibitory concentration $\left(\mathrm{IC}_{50}\right)$ values were calculated using GraphPad Prism 8 (GraphPad Software Inc., USA).

\section{In vivo inhibitory effect of NQP on B. rodhaini}

Fifteen BALB/c mice intraperitoneally challenged with $10^{7}$ B. rodhaini (iRBCs) were randomly assigned to groups ( $n=5$ per group). When parasitemia was about $3 \%$ to $5 \%$, the drug treatment was initiated. The first group received $40 \mathrm{mg} / \mathrm{kg}$ of NQP orally for 5 consecutive days while the second group received 20 $\mathrm{mg} / \mathrm{kg}$ of TAF orally for one time [10-12, 21]. The control group received 5\% DMSO in Milli-Q water. Parasitemia was calculated by counting infected RBCs among 3000 RBCs using Giemsa-stained blood smears. Hematocrit (HCT) changes was monitored as an index for the development of anemia by using hematology analyzer (Nihon MEK-6450, Nihon Kohden Corporation, Tokyo, Japan) every 2 days. On day 42 post infection, all survived mice were euthanized. All animal experiments were approved by the Animal Welfare Committee (approval no. 20-128) and were conducted in accordance with the standards for the care and management of experimental animals as stipulated by the Obihiro University of Agriculture and Veterinary Medicine, Hokkaido, Japan.

\section{Statistical analysis}

Data analysis was performed using GraphPad Prism. Differences in parasitemia between the control and treated groups were determined by one-way ANOVA analysis plus Tukey-Kramer post hoc analysis. Survival rates were calculated using the Kaplan-Meier method, with regard to the log-rank test. A $P$ value $<0.05$ was considered statistically significant.

\section{Results}




\section{NQP inhibits B. gibsoni growth in vitro}

The inhibitory effect of NQP was carried out using in vitro cultured B. gibsoni. NQP significantly inhibited $(P<0.05)$ parasite growth at $2.5 \mu \mathrm{M}$ and showed inhibition in a dose-dependent manner. The $I_{50}$ value of NQP on B. gibsoni was $3.3 \pm 0.5 \mu \mathrm{M}$. Meanwhile, the $\mathrm{IC}_{50}$ value of the control drug TAF was $20.0 \pm 2.4 \mu \mathrm{M}$ (Fig. S1). The results indicate that NQP has potential efficacy against Babesia parasite growth.

\section{In vivo effects of NQP on Babesia infection in mouse model}

The B. rodhaini-infected model was used to further evaluate the effects of NQP on Babesia parasite in vivo. The highest parasitemia of $B$. rodhaini infected mice in DMSO treated group was $78.2 \%$ and all mice died within 10 days post infection (dpi). In contrast, the parasitemia in NQP- and TAF-treated group were significantly $(P<0.05)$ decreased after treatment and showed $95.1 \%$ (3.8\% peak parasitemia) and $95.8 \%$ (3.3\% peak parasitemia) inhibition compared to the DMSO-treated group, respectively. Parasitemia was undetectable via Giemsa-staining method in mice treated with NQP and TAF at $10 \mathrm{dpi}$ and $8 \mathrm{dpi}$, respectively. However, regrowth of parasites was observed in both NQP- $(n=2 / 5)$ and TAF- $(n=1 / 5)$ treated group on $16 \mathrm{dpi}$ and $22 \mathrm{dpi}$ (Fig. 1a). HCT change as an index for anemia development was monitored. Significant $(P<0.05)$ reductions were observed in DMSO-treated group from $6 \mathrm{dpi}$ compared with the values recorded from the NQP- or TAF-treated group (Fig. 1b). All the mice in NQP- and TAF-treated group survived by $40 \mathrm{dpi}(P=0.0002$, Fig. 1c). The Giemsa-stained blood smears of DMSOtreated group from $4 \mathrm{dpi}$ to $7 \mathrm{dpi}$ (8 and $9 \mathrm{dpi}$ are not available), NQP-treated group and TAF-treated group from 4 dpi to 8 dpi are shown in Fig. 2. Compared to the DMSO-treated group, TAF-treated group showed an aberrant parasite phenotype and only faint chromatin staining was visible within 2 days of TAF treatment. Unlike the TAF-treated group, NQP treatment showed a significant degeneration of parasites at 8 dpi.

\section{Discussion}

Because of the increasing number of treatment failures and toxic effects by using current therapies for babesiosis, exploration and discovery of novel drugs with anti-Babesia activity is needed [3, 4, 22]. Babesia and Plasmodium share similar features in their biology as they are closely related genera among the apicomplexan parasites [23]. Due to this, some anti-Babesia drugs were initially investigated because of their antimalarial activity. Several showed excellent activities against Babesia, such as atovaquone combined with azithromycin for human babesiosis, and TAF, which was recently investigated for human and canine babesiosis $[10,24,25]$.

NQ is a 4-aminoquinoline antimalarial drug with a longer half-life but slower action than ART, and is currently combined with ART to treat malaria [14]. This combination therapy also inhibits Schistosoma mansoni [26]. Moreover, NQP combined with azithromycin for malaria treatment was also developed 
[27]. The toxic effects of NQP on mammalian host have been reported. Daily treatment in dogs for 14 days at a dose of $17.5 \mathrm{mg} / \mathrm{kg} /$ day and in rats for $70 \mathrm{mg} / \mathrm{kg} /$ day were considered safe. The safe doses in canine and rat models are equivalent to approximately $10 \mathrm{mg} / \mathrm{kg} /$ day in humans [15, 28]. A previous study reported that the concentration of NQP reached the peak level in plasma 2 hours post treatment with a dose of $10 \mathrm{mg} / \mathrm{kg}$. The peak concentrations were $300.84 \mathrm{ng} / \mathrm{mL}$ and $273.29 \mathrm{ng} / \mathrm{mL}$ in plasma and erythrocytes, respectively, and the half-life of NQP was 198 hours ( 8 days) in normal mice [29]. Interestingly, the concentrations were far greater in $P$. berghei-infected mice [29]. The anti-parasitic activity of NQP as demonstrated by the inhibition of B. gibsoni in vitro (Fig. S1) prompted us to further explore its anti-Babesia activity in vivo. We used the lethal species $B$. rodhaini in the mouse model for evaluating NQP as a therapeutic. In the current in vivo trial, NQP exhibited excellent inhibitory efficacy as evidenced by reduced parasite growth (Fig. 1a) and degenerative morphological changes in the parasites (Fig. 2). Furthermore, the first 2 days of treatment with $40 \mathrm{mg} / \mathrm{kg}$ NQP prevented the rise of $B$. rodhaini parasitemia starting from day 6 post infection compared to the typical rise of mean parasitemia in DMSO-treated mice. In addition, the accumulation of NQP in plasma [29] by completion of the 4-day treatment resulted in morphological changes of parasites in all treated mice at day 8 post infection. Moreover, the TAF-treated group showed an aberrant parasite phenotype (Fig. 2) which has been associated with oxidative stress [10]. B. rodhaini-infected mice in DMSO-treated group developed rapid anemia, whereas NQP and TAF prevented anemia development in infected mice (Fig. 1b).

Since TAF was approved by the U.S. Food and Drug Administration (FDA) as a single drug treatment for malaria, TAF studies have attracted much attention $[10,11]$. Nonetheless, the limitation of TAF is the risk of inducing severe hemolytic anemia in individuals with G6PD deficiency in humans and the relapse of parasites, which are well documented $[10,24,30]$. Moreover, a single treatment of TAF on immunocompromised hosts could not eliminate parasites $[10,24]$. Recently, TAF showed strong and broad anti-parasitic activity against Babesia spp., including $B$. microti, B. gibsoni, and $B$. rodhaini [10]. Hence, TAF was selected as a reference drug in this study. In the present study, the relapse of parasites was observed both in NQP-treated group and TAF-treated group (Fig. 1a). Therefore, NQP may need to be accompanied by other anti-Babesia drugs to augment its effect and prevent the regrowth of parasites.

In addition, the mechanism of action of NQP has not been fully elucidated. The inhibition activity of NQP for Plasmodium was hypothesized to be through the inhibition of hemozoin biocrystallization in the digestive vacuole of late-stage parasites and disruption of membrane system. Due to Babesia not producing hemozoin during parasite development, the inhibitory effect of NQP on the Babesia parasite is hypothesized to be related to targeting the parasite's membrane system [15, 31].

It should be noted that there are some limitations to the present study. Although NQP exhibited a potential antibabesial effect, it has a slower onset of action and a longer half-life, which may easily lead to drug build-up with increasing the probability of developing resistance. Therefore, future studies are warranted to analyze the possible synergistic effect of NQP when administrated in combination with other drug which has a rapid onset of babesicidal action and a short half-life. Such analysis will help to determine 
the most effective composition ratio for treatment of Babesia in animals in clinical applications.

Furthermore, the mode of action by which NQP inhibits the in vitro and in vivo growth of Babesia is still unknown. Subsequently, further studies are required to elucidate this point. Although the present study demonstrated the potential antibabesial efficacy of NQP in a mouse model, additional in vivo experiments are required to confirm such inhibitory effect in B. gibsoni- infected dog.

\section{Conclusion}

The present study demonstrated the inhibitory effect of NQP against $B$. gibsoni in vitro and $B$. rodhaini in vivo. Our findings indicate that NQP is a potential candidate for babesiosis treatment and suggest further investigation on the possible use of this chemical for human babesiosis.

\section{Abbreviations}

NQ: naphthoquine; NQP: naphthoquine phosphate; ART: artemisinin; TAF: tafenoquine; SG1: SYBR Green l; DMSO: dissolved in dimethyl sulfoxide; RBCs: red blood cells; iRBCs: infected red blood cells; i.p.: intraperitoneal; $\mathrm{IC}_{50}$ : half inhibitory concentration; SD: standard deviation; dpi: days post infection; HCT: hematocrit.

\section{Declarations}

\section{Ethics approval and consent to participate}

All procedures were carried out according to the ethical guidelines approved by the Obihiro University of Agriculture and Veterinary Medicine (permit numbers: animal experiment, 20-128; DNA experiment, 1723-4 and 1724-4; pathogen, 201712-5).

\section{Consent for publication}

Not applicable

\section{Availability of data and material}

All data sets were presented as tables, figures and text description in this article.

\section{Competing interests}

The authors declare that they have no competing interests.

\section{Funding}

This study was supported by a Grant-in-Aid for Scientific Research (18H02336 and 18KK0188) and Japan Society for the Promotion of Science Core-to-Core program, both from the Ministry of Education, Culture, Sports, Science, and Technology of Japan, and a grant from the Strategic International 
Collaborative Research Project (JPJ008837) promoted by the Ministry of Agriculture, Forestry and Fisheries of Japan.

\section{Authors' contributions}

SJ and XX designed the study. SJ carried out the experiments. JL, IZ and YH contributed reagents/materials preparation. SJ, ML, MAR, BT and EMG wrote the manuscript and all authors read and approved the final manuscript.

\section{Acknowledgements}

Not applicable

\section{References}

1. Homer MJ, Aguilar-Delfin I, Telford SR, Krause PJ, Persing DH. Babesiosis. Clin Microbiol Rev. 2000;13:451-69.

2. Kavanaugh MJ, Decker CF. Babesiosis. Dis Mon. 2012;58(6):355-60.

3. Vial HJ, Gorenflot A. Chemotherapy against babesiosis. Vet Parasitol. 2006;138:147-60.

4. Mosqueda J, Olvera-Ramirez A, Aguilar-Tipacamu G, Canto GJ. Current advances in detection and treatment of babesiosis. Curr Med Chem. 2012;19:1504-18.

5. Simon MS, Westblade LF, Dziedziech A, Visone JE, Furman RR, Jenkins SG, et al. Clinical and molecular evidence of atovaquone and azithromycin resistance in relapsed Babesia microti infection associated with rituximab and chronic lymphocytic leukemia. Clin Infect Dis. 2017;65:1222-25.

6. Vannier EG, Diuk-Wasser MA, Ben Mamoun C, Krause PJ. Babesiosis. Infect Dis Clin North Am. 2015;29:357-70.

7. White DJ, Talarico J, Chang HG, Birkhead GS, Heimberger T, Morse DL. Human babesiosis in New York State: Review of 139 hospitalized cases and analysis of prognostic factors. Arch Intern Med. 1998;158:2149-54.

8. Gray EB, Herwaldt BL. Babesiosis Surveillance - United States, 2011-2015. MMWR Surveill Summ. 2019;68:1-11.

9. Iguchi A, Soma T, Xuan X. Further epidemiological survey for atovaquone resistant related gene of Babesia gibsoni in Japan during 2015-2018. J Vet Med Sci. 2020;82:1700-3.

10. Liu M, Ji S, Kondoh D, Galon EM, Li J, Tomihari M, et al. Tafenoquine is a promising drug candidate for the treatment of babesiosis. Antimicrob Agents Chemother. 2021;65:e0020421.

11. Carvalho L, Tuvshintulga B, Nugraha AB, Sivakumar T, Yokoyama N. Activities of Artesunate-based Combinations and Tafenoquine Against Babesia bovis In Vitro and Babesia microti In Vivo. Parasit. Vectors. 2020;13:362.

12. Tuvshintulga B, AbouLaila M, Davaasuren B, Ishiyama A, Sivakumar T, Yokoyama N, et al. Clofazimine inhibits the growth of Babesia and Theileria parasites in vitro and in vivo. Antimicrob 
Agents Chemother. 2016;60:2739-46.

13. Rizk MA, El-Sayed SAE, AbouLaila M, Eltaysh R, Yokoyama N, Igarashi I. Performance and consistency of a fluorescence-based high-throughput screening assay for use in Babesia drug screening in mice. Sci. Rep. 2017;7:12774.

14. Wang J, Cao W, Shan C, Zhang M, Li G, Ding D, et al. Naphthoquine phosphate and its combination with artemisinine. Acta Trop. 2004;89:375-81.

15. Moore BR, Laman M, Salman S, Batty KT, Page-Sharp M, Hombhanje F, et al. Naphthoquine: an emerging candidate for artemisinin combination therapy. Drugs. 2016;76:789-804.

16. Hombhanje FW, Huang Q. Artemisinin-naphthoquine combination $\left(\mathrm{ARCO}^{\circledR}\right)$ : an overview of the progress. Pharmaceuticals (Basel). 2010;3:3581-93.

17. Rizk MA, Ji S, Liu M, El-Sayed SAE, Li Y, Byamukama B, et al. Closing the empty anti-Babesia gibsoni drug pipeline in vitro using fluorescence-based high throughput screening assay. Parasitol Int. 2020;75:102054.

18. Sunaga F, Namikawa K, Kanno Y. Continuous in vitro culture of erythrocytic stages of Babesia gibsoni and virulence of the cultivated parasite. J Vet Med Sci. 2002;64:571-5.

19. Terkawi MA, Zhang G, Jia H, Aboge G, Goo YK, Nishikawa Y, et al. C3 contributes to the crossprotective immunity induced by Babesia gibsoni phosphoriboprotein $\mathrm{P} 0$ against a lethal $B$. rodhaini infection. Parasite Immunol. 2008;30:365-70.

20. Ji S, Liu M, Galon EM, Rizk MA, Li J, Li Y, et al. In Vitro Screening of Novel Anti-Babesia gibsoni Drugs from Natural Products. Parasitol. Int. 2021;85:102437.

21. El-Sayed SAE, Rizk MA, Yokoyama N, Igarashi I. Evaluation of the in vitro and in vivo inhibitory effect of thymoquinone on piroplasm parasites. Parasit. Vectors. 2019;12:37.

22. Vannier E, Gewurz BE, Krause PJ. Human babesiosis. Infect Dis Clin North Am. 2008;22:469-88.

23. DeBarry JD, Kissinger JC. Jumbled genomes: missing Apicomplexan synteny. Mol Biol Evol. 2011;28:2855-71.

24. Mordue DG, Wormser GP. Could the drug tafenoquine revolutionize treatment of Babesia microti infection? J Infect Dis. 2019; 220:442-7.

25. Wittner M, Lederman J, Tanowitz HB, Rosenbaum GS, Weiss LM. Atovaquone in the treatment of Babesia microti infections in hamsters. Am J Trop Med Hyg. 1996;55:219-22.

26. El-Beshbishi SN, Taman A, El-Malky M, Azab MS, El-Hawary AK, El-Tantawy DA. First insight into the effect of single oral dose therapy with artemisinin-naphthoquine phosphate combination in a mouse model of Schistosoma mansoni infection. Int J Parasitol. 2013;43:521-30.

27. Bei Z, Li G, Zhao J, Zhang M, Ji X, Wang J. Evaluation of the combination of azithromycin and naphthoquine in animal malaria models. Antimicrob Agents Chemother. 2020;64:e02307-19.

28. Wang J, Zuo B, Xu Z, Sun G, Zhang M, Wang C, et al. Long-term toxicity of co-naphthoquine in beagle dogs. Bull Acad Mil Med Sci. 2003;27:196-8. 
29. Li F, Zhang Z. Artemisinin-Naphthoquine Phosphate Combination (ARCO). In Li G, Li Y, Li Z, Li M, Zeng M, editor. 483-569. Artemisinin-Based and Other Antimalarials, Academic Press; 2018. p 483569.

30. Peters AL, Van Noorden CJ. Glucose-6-phosphate dehydrogenase deficiency and malaria: cytochemical detection of heterozygous G6PD deficiency in women. J Histochem Cytochem. 2009;57:1003-11.

31. Rudzinska MA, Trager W, Lewengrub SJ, Gubert E. An electron microscopic study of Babesia microti invading erythrocytes. Cell Tissue Res. 1976;169:323-34.

\section{Figures}


b
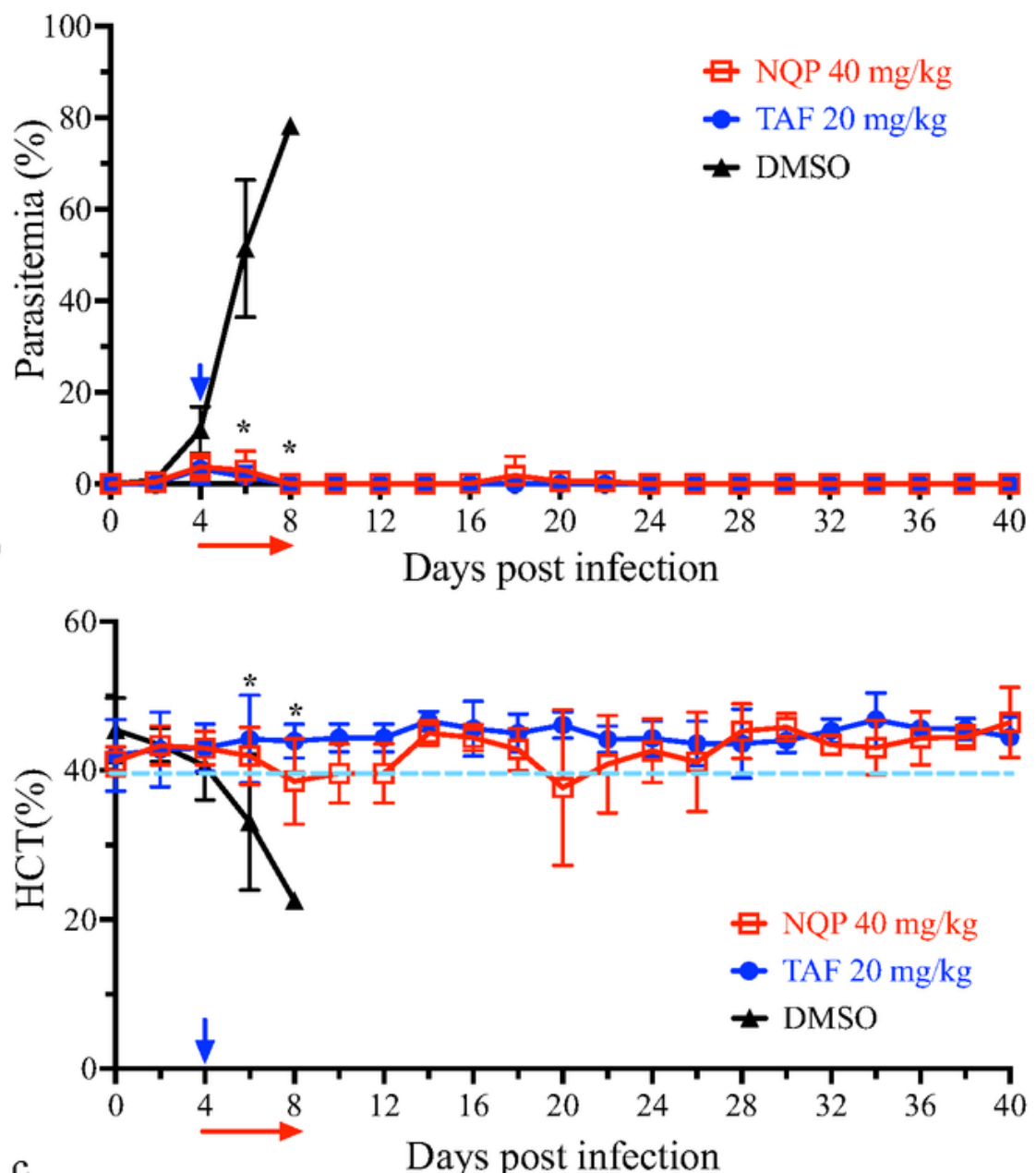

$\mathrm{c}$

Days post infection

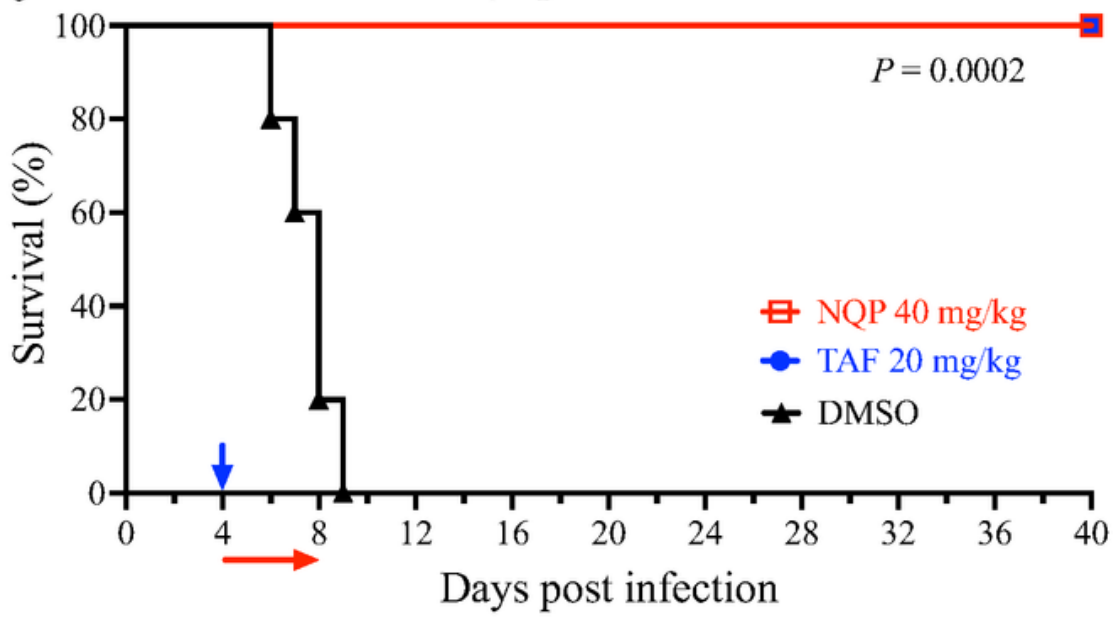

Figure 1

The inhibitory effects of NQP on B. rodhaini in BALB/c mice. (a) The growth inhibition of NQP and TAF as compared with the untreated group. (b) Changes of hematocrit values in mice treated with NQP or TAF as compared with the DMSO-treated group. The asterisks indicate a significant difference $(P<0.05)$ between the NQP- or TAF-treated group and the DMSO-treated group. The arrows indicate time of treatment. (c) Survival rates of NQP and TAF after treatment. The asterisks indicate a significant difference $(P<0.05)$ 
between the NQP- or TAF-treated group and DMSO-treated group. The arrows indicate time of treatment. Parasitemia was calculated by counting infected RBCs among 3000 RBCs using Giemsa-stained blood smears. Dotted line indicates reference range.
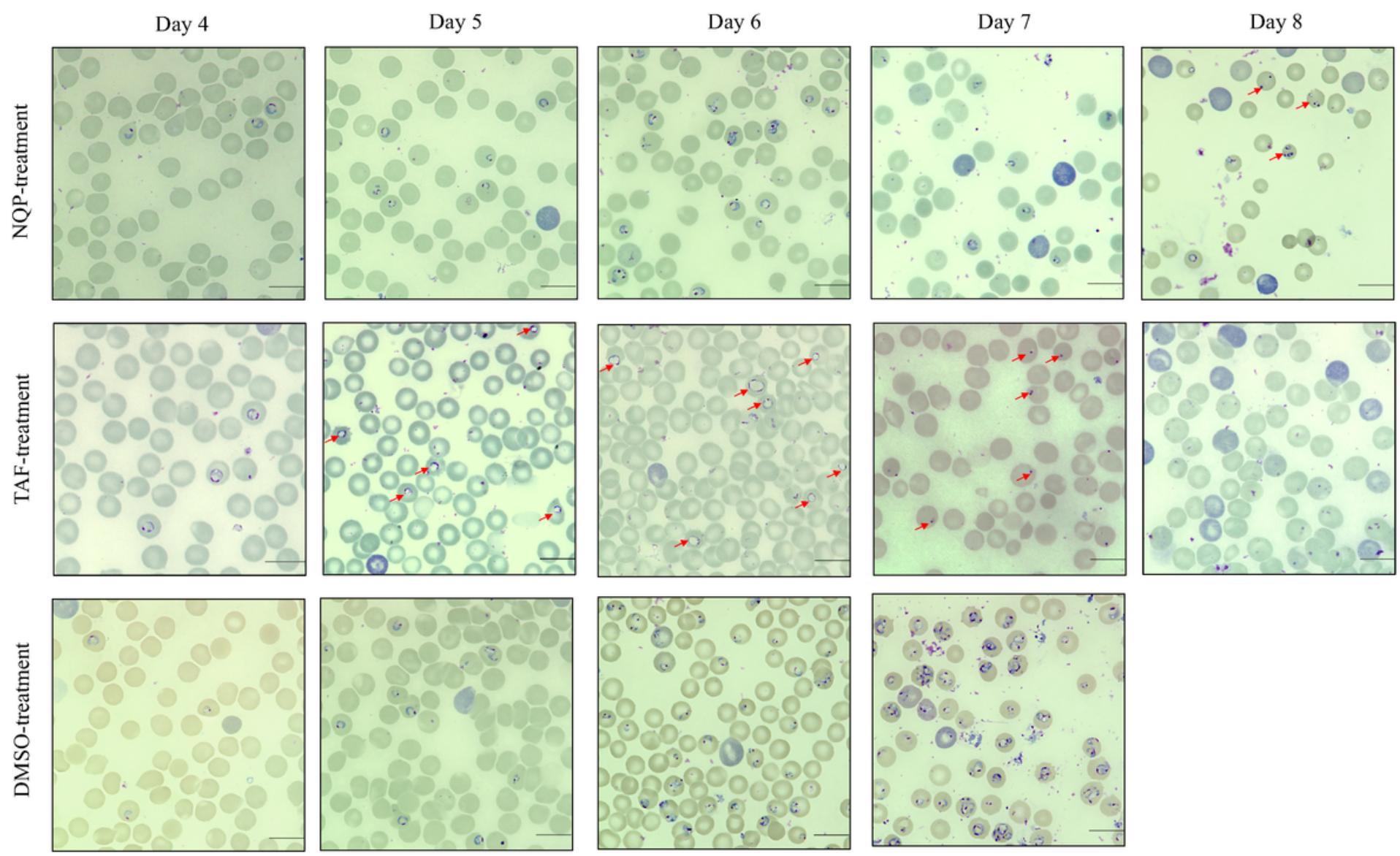

\section{Figure 2}

Light micrographs of B. rodhaini-infected mice during NQP and TAF treatment (from 4 to $8 \mathrm{dpi}$ ) and DMSO treatment (from 4 to $7 \mathrm{dpi}$ ). Compared with the DMSO-treated group, NQP treatment exhibits degenerated parasites at $8 \mathrm{dpi}$ (red arrow), whereas parasites in the TAF-treated group show a vacuolelike aberrant phenotype. Bars $=10 \mu \mathrm{m}$.

\section{Supplementary Files}

This is a list of supplementary files associated with this preprint. Click to download.

- S1.pdf

- Graphabstract.png 